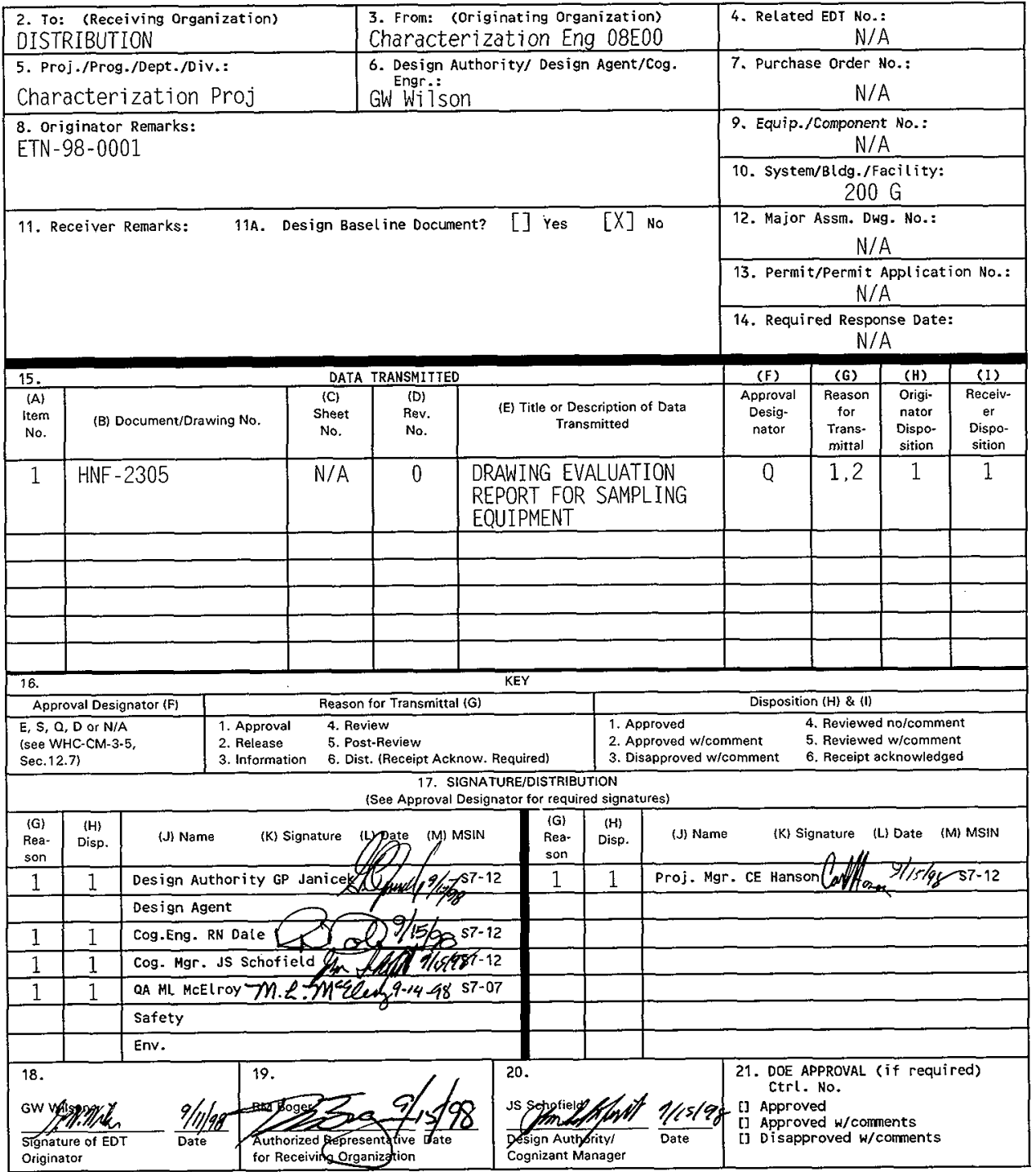




\section{DRAWING EVALUATION REPORT FOR SAMPLING EQUIPMENT DRAWINGS}

G.W. Wilson

COGEMA Engineering Corp., Richland, WA 99352

U.S. Department of Energy Contract DE-AC06-96RL13200

$\begin{array}{lll}\text { EDT/ECN: } & 623329 & \text { UC: } 2070 \\ \text { Org Code: } & 08 E 00 & \text { Charge Code: } \\ \text { B\&R Code: } & \text { EW3120074 } & \text { Tota1 Pages: } 38\end{array}$

Key words: SAMPLING, DRAWING EVALUATION, CATEGORY, RMCS

Abstract: This report documents the results of an evaluation of Characterization sampling equipment drawings for compliance with existing procedures on establishing drawing categories.

TRADEMARK DISCLAIMER. Reference herein to any specific commercial product, process, or service by trade name, trademark, manufacturer, or otherwise, does not necessarily constitute or imply its endorsement, recomendation, or favoring by the United States Government or any agency thereof or its contractors or subcontractors.

Printed in the United States of America. To obtain copies of this document, contact: Document Control Services, P.O. Box 950, Mailstop H6-08, Richland WA 99352, Phone (509) 372-2420; Fax (509) 376-4989.
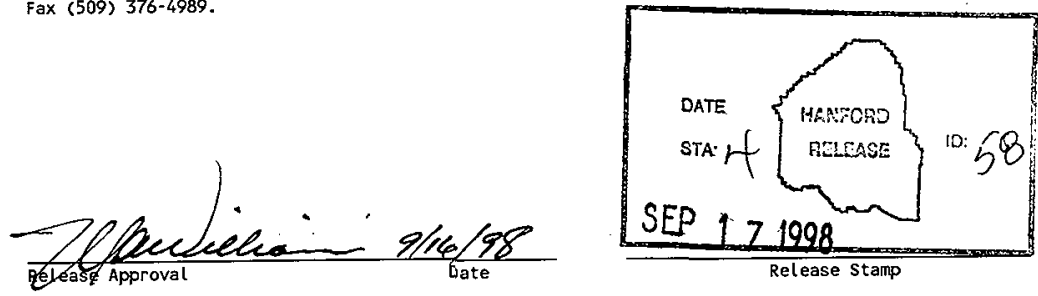

Approved for Public Release 
HNF-2305

Rev. 0

DRAWING EVALUATION REPORT

FOR SAMPLING EQUTPMENT

Prepared for Characterization Engineering by

G. W. Wilson

COGEMA ENGINEERING CORPORATION

September 1998 
HNF-2305

Rev. 0

\section{CONTENTS}

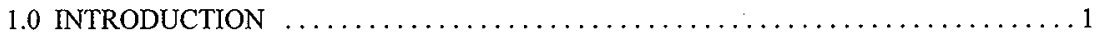

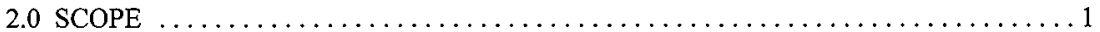

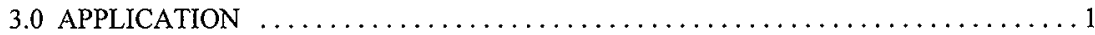

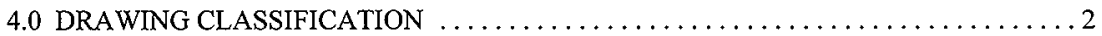

4.1 CORE SAMPLE TRUCK 1 DRAWINGS $\ldots \ldots \ldots \ldots \ldots \ldots \ldots \ldots \ldots$

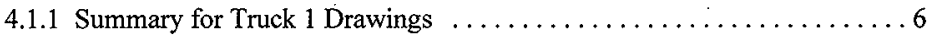

4.2 CORE SAMPLE TRUCK 1 and 2 DRAWINGS $\ldots \ldots \ldots \ldots \ldots \ldots \ldots \ldots \ldots \ldots$

4.2.1 Summary for Truck $1 \& 2$ Drawings $\ldots \ldots \ldots \ldots \ldots \ldots \ldots \ldots$

4.3 CORE SAMPLE TRUCK 2 DRAWINGS $\ldots \ldots \ldots \ldots \ldots \ldots \ldots$

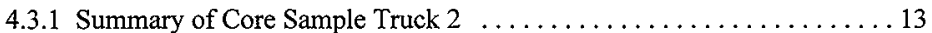

4.4 CORE SAMPLE TRUCK 3 \& 4 DRAWINGS $\ldots \ldots \ldots \ldots \ldots \ldots \ldots \ldots$

4.4.1 Summary RMCST $3 \& 4$ Drawings ...................... 19

4.5 CORE SAMPLING SUPPORT EQUIPMENT DRAWINGS $\ldots \ldots \ldots \ldots \ldots$

4.5.1 Summary for Core Sampling Support Equipment Drawings . . . . . . 27

4.6 RMCST SAMPLING EXHAUSTER DRAWINGS $\ldots \ldots \ldots \ldots \ldots \ldots \ldots 27$

4.6.1 Summary for RMCST Exhauster Drawings $\ldots \ldots \ldots \ldots \ldots \ldots \ldots . \ldots \ldots$

4.7 OTHER SAMPLING EQUIPMENT DRAWINGS $\ldots \ldots \ldots \ldots \ldots \ldots \ldots$

4.7.1 Summary for Other Sampling Equipment Drawings .......... 33

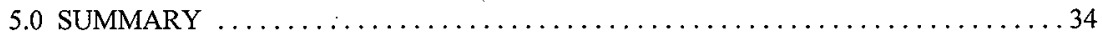

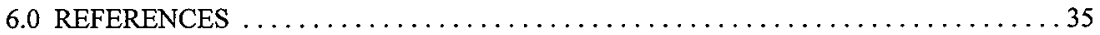


HNF-2305

Rev. 0

\subsection{INTRODUCTION}

This document presents the results of a task to evaluate Tank Waste Remediation System (TWRS) sampling equipment drawings and identifies drawings category as either essential, support, or general drawings. This report completes the drawing evaluation task as outlined in Engineering Task Plan For Truck 3 \& 4 Drawing Compliance and Evaluation (Wilson, 1997).

\subsection{SCOPE}

The scope of this report is limited to an evaluation and identification of drawing category for drawings of certain tank waste sampling equipment for which the TRWS Characterization Project has been assigned custody, including: vapor sampling, grab sampling, auger sampling, and all core sampling equipment (see LMHC Task Order 304). This report does not address drawings for other waste tank deployed equipment systems having similar assigned custody, such as, Light Duty Utility Arm (LDUA), Cone Penetrometer system, or Long Length Contaminated Equipment (LLCE). The LDUA drawings are addressed in the Characterization Equipment Essential Drawings (HNF 1998). The Cone Penetrometer system drawings which are vendor drawings (not $\mathrm{H}$ - series) is not currently turned over to operations for deployment. The LLCE equipment was just recently assigned to Characterization Project and were not included in the original scope for this evaluation and will be addressed in the evaluation update scheduled for fiscal year 1999.

\subsection{APPLICATION}

Engineering Drawing Requirements (HNF 1997) defines the drawing categories as follows.

a. Essential - Drawings necessary to directly support the safe operation or maintenance of the facility.

b. Support - Drawings that further describe the design details of structure, systems, of components shown of essential drawings or are frequently used by the support staff. 
HNF-2305

Rev. 0

c. General - Drawings that are historical in nature or not frequently used in the operation or maintenance of the facility.

All TWRS sampling equipment is mobile equipment that is not directly associated with any one facility. Therefore, this application implemented the requirements of Engineering Drawings Requirements (HNF 1997) tempered by the fact that this equipment is not a permanent on-line facility.

The drawings identified as generally meeting the description of essential consist of the the following types:

* Drawings that describe equipment required for safe operations (drill string arrangements)

* Drawings that depict flow diagrams (hydraulic, pneumatic, and P\&ID diagrams)

* Drawings that depict electrical connections (elementary and one-line diagrams)

* Drawings that depict electrical power connection (one-line and connection diagrams)

\subsection{DRAWING CLASSIFICATION}

The following sections are a detailed listing of the drawings that were evaluated and assigned drawing category. The listings shows the drawing category assigned in the Cat.column with an $\mathrm{E}$ for drawings that are determined to be essential drawings, an $\mathrm{S}$ for support drawing, and a $\mathrm{G}$ for general drawings. For specific information about equipment drawings, refer to the specified section of this document.

Core Sample Truck 1 Drawings ................................ See Section 4.1

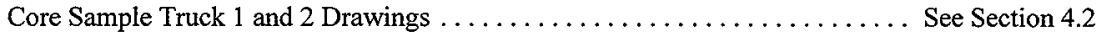

Core Sample Truck 2 Drawings ........................... See Section 4.3

RMCST Truck 3 and 4 Drawings $\ldots \ldots \ldots \ldots \ldots \ldots \ldots \ldots \ldots$ See Section 4.4

Core Sampling Support Equipment Drawings $\ldots \ldots \ldots \ldots \ldots \ldots \ldots$ See Section 4.5

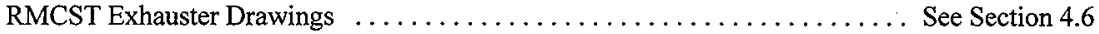

Other Sampling Equipment Drawings $\ldots \ldots \ldots \ldots \ldots \ldots \ldots \ldots \ldots \ldots$ See Section 4.7 
HNF-2305

Rev. 0

\subsection{CORE SAMPLE TRUCK 1 DRAWINGS}

Table 4.1 Core Sample Truck 1 Drawings

\begin{tabular}{|c|c|c|c|c|}
\hline Dwg. No. & Sht. & Description & Cat. & Justification/Notes \\
\hline $\mathrm{H}-2-38075$ & $1-5$ & Shielded Receiver Assembly & $S$ & \\
\hline $\mathrm{H}-2-81790$ & 1 & $\begin{array}{l}\text { Hydrostatic Head Addition } \\
\text { System Flow Diagram }\end{array}$ & G & Installed, not used \\
\hline $\mathrm{H}-2-81832$ & $1-2$ & $\begin{array}{l}\text { Drill Rig Junction Box Enclosure } \\
\text { Assembly }\end{array}$ & G & \\
\hline $\mathrm{H}-2-81833$ & $1-2$ & $\begin{array}{l}\text { Hydraulic Bottom Detector Alarm } \\
\text { Panel Assembly }\end{array}$ & $S$ & \\
\hline H-9-90257 & 1 & $\begin{array}{l}\text { Core Sampler Latch Mechanism } \\
\text { Assembly \& Detail }\end{array}$ & G & Not Used \\
\hline $\mathrm{H}-2-91390$ & $1-2$ & Grapple Hoist Installation & S & $\begin{array}{l}\text { Note: Important for } \\
\text { Maintenance }\end{array}$ \\
\hline $\mathrm{H}-2-91391$ & $1-5$ & Grapple Hoist Assembly & $S$ & $\begin{array}{l}\text { Note: Important for } \\
\text { Maintenance }\end{array}$ \\
\hline H-2-91392 & $1-5$ & Grapple Hoist Box Assembly & $S$ & $\begin{array}{l}\text { Note: Important for } \\
\text { Maintenance }\end{array}$ \\
\hline $\mathrm{H}-2-91393$ & 1 & $\begin{array}{l}\text { Grapple Hoist Box Housing } \\
\text { Assembly }\end{array}$ & G & \\
\hline H-2-91394 & 1 & Encoder Panel Assembly & G & \\
\hline H-2-91395 & 1 & Core Sampler Truck Details & G & Not Used \\
\hline H-2-91397 & $1-4$ & Pressure Readout Display Cabinet & G & \\
\hline $\mathrm{H}-2-91477$ & 1 & Shielded Receiver Assembly & G & Not Used \\
\hline $\mathrm{H}-2-91583$ & 1 & Quill Rod Stop Truck 1 & G & \\
\hline $\mathrm{H}-2-91650$ & 1 & $\begin{array}{l}\text { Core Sampler Truick } 1 \text { Drawing } \\
\text { Index }\end{array}$ & $S$ & \\
\hline $\mathrm{H}-2-91651$ & $1-3$ & Shielded Receiver Assembly & $\mathrm{G}$ & \\
\hline
\end{tabular}


HNF-2305

Rev. 0

Table 4.1 Core Sample Truck 1 Drawings

\begin{tabular}{|c|c|c|c|c|}
\hline Dwg. No. & Sht. & Description & Cat. & Justification/Notes \\
\hline $\mathrm{H}-2-91652$ & 1 & $\begin{array}{l}\text { Shielded Receiver Weather Cover } \\
\text { Assembly }\end{array}$ & $G$ & \\
\hline $\mathrm{H}-2-91653$ & $1-3$ & Sampler Hoist Assembly & $S$ & $\begin{array}{l}\text { Note: Important for } \\
\text { Maintenance }\end{array}$ \\
\hline H-2-91654 & $1-2$ & Sampler Hoist Housing Assembly & $\mathrm{G}$ & \\
\hline $\mathrm{H}-2-91655$ & $1-2$ & $\begin{array}{l}\text { Sampler Hoist Load Stand } \\
\text { Assembly }\end{array}$ & G & \\
\hline$H-2-91656$ & 1 & $\begin{array}{l}\text { Sampler Hoist Cable Wiper } \\
\text { Assembly }\end{array}$ & $\mathrm{G}$ & \\
\hline $\mathrm{H}-2-91657$ & 1 & $\begin{array}{l}\text { Sampler Hoist Cable Drum } \\
\text { Assembly }\end{array}$ & G & \\
\hline $\mathrm{H}-2-91658$ & 1 & $\begin{array}{l}\text { Sampler Hoist Cable Feed } \\
\text { Assembly }\end{array}$ & G & \\
\hline $\mathrm{H}-2-91659$ & 1 & Sampler Hoist Cover Assembly & G & \\
\hline $\mathrm{H}-2-91660$ & 1 & $\begin{array}{l}\text { Shielded Receiver Mount } \\
\text { Assembly }\end{array}$ & $\mathrm{G}$ & \\
\hline $\mathrm{H}-2-91661$ & 1 & $\begin{array}{l}\text { Sampler Hoist Cable Guide } \\
\text { Assembly }\end{array}$ & $\mathrm{G}$ & - \\
\hline $\mathrm{H}-2-91663$ & $1-3$ & Shielded Receiver Base Assembly & G & \\
\hline H-2-91664 & 1 & $\begin{array}{l}\text { Shielded Receiver Tube Section } \\
\text { Assembly }\end{array}$ & G & \\
\hline $\mathrm{H}-2-91686$ & $1-2$ & $\begin{array}{l}\text { Grapple Hoist Rotary Valve } \\
\text { Actuator. }\end{array}$ & $S$ & $\begin{array}{l}\text { Note: Important for } \\
\text { Maintenance }\end{array}$ \\
\hline$H-2=91688$ & 1 & Quill Rod Adapter Truck 1 & G & Not used \\
\hline H-2-91701 & $1-7$ & $\begin{array}{l}\text { Truck Assembly Core Sampler } \\
\text { Truck \#1 }\end{array}$ & $\mathrm{S}$ & $\begin{array}{l}\text { Note: Important for } \\
\text { Maintenance }\end{array}$ \\
\hline
\end{tabular}


HNF-2305

Rev. 0

Table 4.1 Core Sample Truck 1 Drawings

\begin{tabular}{|c|c|c|c|c|}
\hline Dwg. No. & Sht. & Description & Cat. & Justification/Notes \\
\hline $\mathrm{H}-2-91701$ & 8 & $\begin{array}{l}\text { Truck Assembly Core Sampler } \\
\text { Truck \#1 }\end{array}$ & E & $\begin{array}{l}\text {-Depicts Equipment } \\
\text { Essential to Safe Operation } \\
\text { (Z-Purge Diagram) }\end{array}$ \\
\hline $\mathrm{H}-2-91701$ & $9-10$ & $\begin{array}{l}\text { Truck Assembly Core Sampler } \\
\text { Truck \#1 }\end{array}$ & $\mathrm{S}$ & $\begin{array}{l}\text { Note: Important for } \\
\text { Maintenance }\end{array}$ \\
\hline $\mathrm{H}-2-91714$ & $1-2$ & $\begin{array}{l}\text { Drill Head Service Platform \#1 } \\
\text { Assembly }\end{array}$ & G & . \\
\hline $\mathrm{H}-2-91719$ & $1-3$ & Hydraulic Installation & $S$ & $\begin{array}{l}\text { Note: Important for } \\
\text { Maintenance }\end{array}$ \\
\hline $\mathrm{H}-2-91720$ & $1-3$ & $\begin{array}{l}\text { Core Sampler Truck \#1 Hydraulic } \\
\text { Flow Diagram }\end{array}$ & $\mathrm{E}$ & $\begin{array}{l}\text {-Depicts High-Pressure } \\
\text { Process Diagram }\end{array}$ \\
\hline $\mathrm{H}-2-91721$ & $1-3$ & Drillhead Hydraulic Piping Install & $\mathrm{S}$ & $\begin{array}{l}\text { Note: Important for } \\
\text { Maintenance }\end{array}$ \\
\hline $\mathrm{H}-2-91722$ & $1-2$ & $\begin{array}{l}\text { Grapple \& Grapple Hoist } \\
\text { Installation }\end{array}$ & G & Not Used \\
\hline $\mathrm{H}-2-91723$ & $1-4$ & Grapple Hoist Assembly & G & Not Used \\
\hline $\mathrm{H}-2-91724$ & $1-5$ & Grapple Box Assembly & $\mathrm{G}$ & Not Used \\
\hline $\mathrm{H}-2-91725$ & 1 & Grapple Box Housing Assembly & $\mathrm{G}$ & Not Used \\
\hline $\mathrm{H}-2-92264$ & 1 & $\begin{array}{l}\text { Control and Power Cable } \\
\text { Assembly }\end{array}$ & $\mathrm{G}$ & \\
\hline H-2-92315 & 1 & $\begin{array}{l}\text { Hydraulic and Sampler Actuator } \\
\text { Control Panel }\end{array}$ & G & \\
\hline $\mathrm{H}-2-92420$ & $1-3$ & Electrical Installation & $\mathrm{S}$ & $\begin{array}{l}\text { Note: Important for } \\
\text { Maintenance }\end{array}$ \\
\hline $\mathrm{H}-2-92421$ & $1-4$ & Control Console Assembly & $S$ & $\begin{array}{l}\text { Note: Important for } \\
\text { Maintenance }\end{array}$ \\
\hline
\end{tabular}


HNF-2305

Rev. 0

Table 4.1 Core Sample Truck 1 Drawings

\begin{tabular}{|c|c|c|c|c|}
\hline Dwg. No. & Sht. & Description & Cat. & Justification/Notes \\
\hline H-2-92422 & $1-6$ & $\begin{array}{l}\text { Core Sample Truck \#1 Connection } \\
\text { Diagram }\end{array}$ & $\mathrm{E}$ & $\begin{array}{l}\text {-Depicts Equipment } \\
\text { Essential to Safe } \\
\text { Operation, -Depicts } \\
\text { Isolation Boundary for } \\
\text { Electrical or High-Pressure } \\
\text { Fluid Systems }\end{array}$ \\
\hline $\mathrm{H}-2-92432$ & $1-2$ & $\begin{array}{l}\text { Shield Receiver Electrical } \\
\text { Installation }\end{array}$ & $\mathrm{S}$ & $\begin{array}{l}\text { Note: Important for } \\
\text { Maintenance }\end{array}$ \\
\hline H-2-92433 & 1 & Panel Assembly - Circuit Breaker & $\mathrm{G}$ & \\
\hline $\mathrm{H}-2-92434$ & 1 & Panel Detail -Circuit Breaker & $\mathrm{G}$ & \\
\hline H-2-99726 & $1-2$ & $\begin{array}{l}\text { Shielded Receiver Tube } \\
\text { Replacement }\end{array}$ & $G$ & \\
\hline H-2-827186 & $1-3$ & $\begin{array}{l}\text { Electrical Truck } 1 \text { Connection } \\
\text { Diagram }\end{array}$ & $\mathrm{E}$ & $\begin{array}{l}\text {-Depicts Electrical Power } \\
\text { Diagrams }\end{array}$ \\
\hline $\mathrm{H}-2-827190$ & 1 & $\begin{array}{l}\text { Electrical Truck } 1 \text { One-Line } \\
\text { Diagram }\end{array}$ & $\mathrm{E}$ & -Depicts Electrical one-line \\
\hline
\end{tabular}

\subsubsection{Summary for Truck 1 Drawings}

Table 4.1.1 Summary for Truck 1 Drawings

\begin{tabular}{|l|c|c|}
\hline \hline \multicolumn{1}{|c|}{ Category } & Drawings & Sheets \\
\hline General Drawings & 32 & 52 \\
\hline Support Drawings. & $14^{*}$ & 49 \\
\hline Essential Drawings. & 5 & 14 \\
\hline
\end{tabular}

*Note: Drawing H-2-91701 is included in the essential drawing total. 
HNF-2305

Rev. 0

\subsection{CORE SAMPLE TRUCK 1 and 2 DRAWINGS}

Table 4.2 Core Sample Truck $1 \& 2$ Drawings

\begin{tabular}{|c|c|c|c|c|}
\hline Dwg. & Sht. & Description & Cat. & Justification/Notes \\
\hline $\mathrm{H}-2-91428$ & $1-2$ & $\begin{array}{l}\text { Shielded Receiver View Port } \\
\text { Assembly }\end{array}$ & G & \\
\hline $\mathrm{H}-2-91665$ & $1-3$ & Remote Latch Unit Assembly & $\mathrm{G}$ & \\
\hline $\mathrm{H}-2-91666$ & 1 & $\begin{array}{l}\text { 3" Ball Valve Modification } \\
\text { Assembly }\end{array}$ & $\mathrm{G}$ & \\
\hline H-2-91667 & 1 & Dust Cap and Sleeve Assembly & G & \\
\hline$H-2-91668$ & 1 & $\begin{array}{l}\text { Remote Latch Unit Weight } \\
\text { Assembly }\end{array}$ & G & Not Used \\
\hline $\mathrm{H}-2-91669$ & 1 & Sampler Hoist Interlock Assembly & $\mathrm{G}$ & \\
\hline $\mathrm{H}-2-91702$ & $1-3$ & $\begin{array}{l}\text { Core Sampler Truck Bed } \\
\text { Assembly }\end{array}$ & G & \\
\hline $\mathrm{H}-2-91703$ & 1 & $\begin{array}{l}\text { Lower Frame \& Rotation Stop } \\
\text { Assembly }\end{array}$ & G & \\
\hline $\mathrm{H}-2-91704$ & 1 & Lower Frame Assembly & G & \\
\hline $\mathrm{H}-2-91705$ & 1 & Lower Bearing Mount Assembly & G & \\
\hline $\mathrm{H}-2-91706$ & $1-2$ & Stationary Platform Assembly & G & \\
\hline $\mathrm{H}-2-91707$ & $1-2$ & Rotating Platform Assembly & $\mathrm{S}$ & $\begin{array}{l}\text { Note: Important for } \\
\text { Maintenance }\end{array}$ \\
\hline $\mathrm{H}-2-91708$ & $1-2$ & $\begin{array}{l}\text { Rotating Platform Weldment } \\
\text { Assembly }\end{array}$ & G & \\
\hline $\mathrm{H}-2-91709$ & 1 & Bearing Plate Detail & $\mathrm{G}$ & \\
\hline $\mathrm{H}-2-91710$ & $1-5$ & $\begin{array}{l}\text { Rotating \& Stationary Platform } \\
\text { Safety Rails Assembly }\end{array}$ & G & \\
\hline $\mathrm{H}-2-91711$ & $1-2$ & $\begin{array}{l}\text { Switch Mounts, Stops \& Brackets } \\
\text { Assembly \& Details }\end{array}$ & $\mathrm{G}$ & \\
\hline
\end{tabular}


HNF-2305

Rev. 0

Table 4.2 Core Sample Truck 1 \& 2 Drawings

\begin{tabular}{|c|c|c|c|c|}
\hline Dwg. & Sht. & Description & Cat. & Justification/Notes \\
\hline $\mathrm{H}-2-91712$ & 1 & Slide Rail Details & $\mathrm{G}$ & \\
\hline H-2-91713 & 1 & Control Console Support Frame & $\mathrm{G}$ & \\
\hline $\mathrm{H}-2-91715$ & 1 & $\begin{array}{l}\text { Shielded Receiver Lifting Frame } \\
\text { Installation }\end{array}$ & G & \\
\hline $\mathrm{H}-2-91716$ & $1-4$ & $\begin{array}{l}\text { Shielded Receiver Lifting Frame } \\
\text { Assembly }\end{array}$ & $\mathrm{G}$ & . \\
\hline $\mathrm{H}-2-91717$ & 1 & Air Compressor Frame Assembly & $\mathrm{G}$ & \\
\hline $\mathrm{H}-2-91718$ & $1-2$ & Rear Jack Assembly & G & \\
\hline $\mathrm{H}-2-92423$ & 1 & Motor Control Chassis Assembly & $\mathrm{G}$ & \\
\hline H-2-92424 & $1-2$ & $\begin{array}{l}\text { Panel and Control Chassis } \\
\text { Assembly }\end{array}$ & G & \\
\hline $\mathrm{H}-2-92427$ & 1 & $\begin{array}{l}\text { Latching Control Chassis } \\
\text { Assembly }\end{array}$ & G & \\
\hline H-2-92428 & $1-2$ & Latching Control Chassis Details & G & \\
\hline H-2-92429 & 1 & Latch Control Panel Assembly & G & \\
\hline $\mathrm{H}-2-92430$ & 1 & $\begin{array}{l}\text { Latch Control Circuit Board } \\
\text { Details }\end{array}$ & $\mathrm{G}$ & . \\
\hline $\mathrm{H}-2-92431$ & 1 & Latch Control Logic Diagram & $\mathrm{S}$ & \\
\hline $\mathrm{H}-2-92435$ & 1 & Vent Panel Assembly & $\mathrm{G}$ & \\
\hline
\end{tabular}


HNF-2305

Rev. 0

\subsubsection{Summary for Truck $1 \& 2$ Drawings}

Table 4.2.1 Summary for Truck $1 \& 2$ Drawings

\begin{tabular}{||c|c|c|}
\hline \multicolumn{1}{|c|}{ Category } & Drawings & Sheets \\
\hline General Drawings & 28 & 46 \\
\hline Support Drawings. & 2 & 3 \\
\hline Essential Drawings. & 0 & 0 \\
\hline
\end{tabular}

\subsection{CORE SAMPLE TRUCK 2 DRAWINGS}

Table 4.3 Core Sample Truck 2 Drawings

\begin{tabular}{||c|c|l|c|l||}
\hline Dwg. & Sht. & \multicolumn{1}{|c|}{ Description } & Cat. & Justification/Notes \\
\hline \hline H-2-131570 & 1 & $\begin{array}{l}\text { Sampler Hoist Cable Drum } \\
\text { Assembly }\end{array}$ & G & \\
\hline H-2-140300 & $1-3$ & Core Sampler Truck 2 Assembly & S & $\begin{array}{l}\text { Note: Important for } \\
\text { Maintenance }\end{array}$ \\
\hline H-2-140301 & $1-2$ & $\begin{array}{l}\text { Drawing Index Core Sampler } \\
\text { Truck 2 }\end{array}$ & S & $\begin{array}{l}\text { Note: Important for } \\
\text { Maintenance }\end{array}$ \\
\hline H-2-140302 & $1-6$ & Drill Rig Equipment Installation & G & \\
\hline H-2-140303 & $1-6$ & Electrical Installation & S & $\begin{array}{l}\text { Note: Important for } \\
\text { Maintenance }\end{array}$ \\
\hline H-2-140304 & $1-2$ & $\begin{array}{l}\text { Drill Rig Junction Box Enclosure } \\
\text { Assembly }\end{array}$ & G & \\
\hline H-2-140305 & $1-2$ & $\begin{array}{l}\text { Hydraulic Bottom Detector Alarm } \\
\text { Panel Assembly \& Details }\end{array}$ & G & \\
\hline H-2-140306 & 1 & Up Limit Switch Proximity Probe & G & \\
\hline H-2-140307 & $1-2$ & Aux Air Supply Installation & G & \\
\hline
\end{tabular}


HNF-2305

Rev. 0

Table 4.3 Core Sample Truck 2 Drawings

\begin{tabular}{|c|c|c|c|c|}
\hline Dwg. & Sht. & Description & Cat. & Justification/Notes \\
\hline $\mathrm{H}-2-140308$ & $1-3$ & Truck Frame Assembly & G & \\
\hline $\mathrm{H}-2-140309$ & $1-2$ & Center Outrigger Jack Assembly & G & \\
\hline $\mathrm{H}-2-81785$ & $1-17$ & $\begin{array}{l}\text { Core Sample Truck \#2 Electrical } \\
\text { Connection Diagram }\end{array}$ & $\mathrm{S}$ & $\begin{array}{l}\text { Note: Important for } \\
\text { Maintenance }\end{array}$ \\
\hline $\mathrm{H}-2-81834$ & $1-5$ & Grapple Hoist Installation & $\mathrm{S}$ & $\begin{array}{l}\text { Note: Important for } \\
\text { Maintenance }\end{array}$ \\
\hline $\mathrm{H}-2-81835$ & $1-2$ & $\begin{array}{l}\text { Grapple Hoist Mounting Support } \\
\text { Assembly }\end{array}$ & G & \\
\hline H-2-81836 & $1-6$ & Grapple Hoist Assembly & $\mathrm{S}$ & $\begin{array}{l}\text { Note: Important for } \\
\text { Maintenance }\end{array}$ \\
\hline $\mathrm{H}-2-81837$ & $1-2$ & Grapple Hoist Housing Assembly & G & . \\
\hline $\mathrm{H}-2-81838$ & $1-4$ & Purge Gas Enclosure Assembly & G & \\
\hline $\mathrm{H}-2-81839$ & $1-5$ & Purge Gas Piping Assembly & $S$ & $\begin{array}{l}\text { Note: Important for } \\
\text { Maintenance }\end{array}$ \\
\hline $\mathrm{H}-2-81840$ & $1-3$ & Control Console Assembly & G & \\
\hline $\mathrm{H}-2-81841$ & $1-6$ & $\begin{array}{l}\text { Purge Gas Piping and Hose } \\
\text { Installation }\end{array}$ & $S$ & $\begin{array}{l}\text { Note: Important for } \\
\text { Maintenance }\end{array}$ \\
\hline H-2-81844 & $1-3$ & Quill Rod Assembly & $\mathrm{G}$ & Not used \\
\hline $\mathrm{H}-2-81845$ & $1-2$ & Kamlock Adapter Assembly & $\mathrm{G}$ & \\
\hline $\mathrm{H}-2-81846$ & $1-3$ & Hydraulic Reservoir Assembly & $\mathrm{G}$ & \\
\hline $\mathrm{H}-2-81848$ & $1-2$ & $\begin{array}{l}\text { Grapple Hoist Rotary Valve } \\
\text { Actuator Assembly }\end{array}$ & $\mathrm{G}$ & \\
\hline H-2-81849 & 1 & Remote Latch Unit Weight Assy & G & \\
\hline $\mathrm{H}-2-81850$ & $1-8$ & Hydraulic Controls Installation & $\mathbf{S}$ & $\begin{array}{l}\text { Note: Important for } \\
\text { Maintenance }\end{array}$ \\
\hline $\mathrm{H}-2-81851$ & 1 & Cable Connection Diagram & G & \\
\hline
\end{tabular}


HNF-2305

Rev. 0

Table 4.3 Core Sample Truck 2 Drawings

\begin{tabular}{|c|c|c|c|c|}
\hline Dwg. & Sht. & Description & Cat. & Justification/Notes \\
\hline $\mathrm{H}-2-81852$ & 1 & $\begin{array}{l}\text { Overall Electrical Power } \\
\text { Distribution Diagram }\end{array}$ & G & $\begin{array}{l}\text { Duplicates information on } \\
\mathrm{H}-2-827187\end{array}$ \\
\hline $\mathrm{H}-2-81854$ & 1 & Loadcell Readout Assembly & $\mathrm{G}$ & \\
\hline $\mathrm{H}-2-81855$ & $1-2$ & Front Jack Assembly & G & \\
\hline $\mathrm{H}-2-82102$ & $1-3$ & $\begin{array}{l}\text { Line Conditioner \& Control Select } \\
\text { Panel Assembly }\end{array}$ & $\mathrm{G}$ & \\
\hline $\mathrm{H}-2-82103$ & 1 & $\begin{array}{l}\text { Line Conditioner \& Control Select } \\
\text { Panel Installation }\end{array}$ & G & \\
\hline $\mathrm{H}-2-821454$ & $1-4$ & Breathing Air Piping Installation & G & $\begin{array}{l}\text { Note: Installed but not } \\
\text { used }\end{array}$ \\
\hline $\mathrm{H}-2-83111$ & $1-7$ & $\begin{array}{l}\text { Core Sampler Truck \#2 } \\
\text { Elementary Diagrams }\end{array}$ & $\mathrm{E}$ & $\begin{array}{l}\text {-Depicts Equipment } \\
\text { Essential to Safe Operation } \\
\text {-Depicts Required Alarm } \\
\text { Systems -Depicts \& } \\
\text { Identifies Instrumentation } \\
\text { Required by AB }\end{array}$ \\
\hline $\mathrm{H}-2-83112$ & $1-6$ & Instrument Enclosure Assembly & $\mathrm{S}$ & $\begin{array}{l}\text { Note: Important for } \\
\text { Maintenance }\end{array}$ \\
\hline $\mathrm{H}-2-83115$ & $1-4$ & Purge Gas Assembly & $\mathrm{S}$ & $\begin{array}{l}\text { Note: Important for } \\
\text { Maintenance }\end{array}$ \\
\hline $\mathrm{H}-2-83307$ & $1-5$ & Sampler Hoist Assembly & $\mathrm{S}$ & $\begin{array}{l}\text { Note: Important for } \\
\text { Maintenance }\end{array}$ \\
\hline $\mathrm{H}-2-83308$ & $1-3$ & Sampler Hoist Housing Assembly & $\mathrm{G}$ & \\
\hline H-2-83309 & $1-2$ & Shielded Receiver Base Assembly & $\mathrm{G}$ & \\
\hline $\mathrm{H}-2-83310$ & $1-2$ & Sampler Hoist Load Stand & G & \\
\hline $\mathrm{H}-2-83314$ & $1-4$ & Shielded Receiver Assembly & $\mathrm{s}$ & $\begin{array}{l}\text { Note: Important for } \\
\text { Maintenance }\end{array}$ \\
\hline
\end{tabular}


HNF-2305

Rev. 0

Table 4.3 Core Sample Truck 2 Drawings

\begin{tabular}{|c|c|c|c|c|}
\hline Dwg. & Sht. & Description & Cat. & Justification/Notes \\
\hline $\mathrm{H}-2-83315$ & $1-2$ & $\begin{array}{l}\text { Shielded Receiver Electrical } \\
\text { Assembly }\end{array}$ & $\mathrm{S}$ & $\begin{array}{l}\text { Note: Important for } \\
\text { Maintenance }\end{array}$ \\
\hline $\mathrm{H}-2-83316$ & $1-3$ & $\begin{array}{l}\text { Shielded Receiver Weather Cover } \\
\text { Assembly }\end{array}$ & G & \\
\hline $\mathrm{H}-2-83391$ & $1-3$ & Hydraulic Flow Diagram & $\mathrm{E}$ & -Depicts Process Diagrams \\
\hline $\mathrm{H}-2-85068$ & $1-2$ & Purge Gas Pneumatic Diagram & $\mathrm{E}$ & $\begin{array}{l}\text {-Depicts High Pressure } \\
\text { Process \& Instrumentation } \\
\text { Diagram }\end{array}$ \\
\hline H-2-89435 & $1-4$ & $\begin{array}{l}\text { Drillhead Service Platform } \\
\text { Installation }\end{array}$ & G & \\
\hline H-2-89436 & $1-3$ & $\begin{array}{l}\text { Drillhead Service Platform } \\
\text { Assembly }\end{array}$ & G & \\
\hline H-2-89437 & $1-2$ & Rpm Sensing Ring Installation & $\mathrm{G}$ & \\
\hline $\mathrm{H}-2-89438$ & $1-4$ & Penetration Rate Assembly & G & \\
\hline $\mathrm{H}-2-89439$ & $1-5$ & $\begin{array}{l}\text { Drillhead Service Platform } \\
\text { Assembly }\end{array}$ & G & \\
\hline $\mathrm{H}-2-89440$ & $1-2$ & Pressure Indicator Light Assembly & G & \\
\hline $\mathrm{H}-2-89441$ & 1 & Circuit Breaker Panel Detail & G & \\
\hline $\mathrm{H}-2-89442$ & $1-3$ & Drillhead Hydraulic Installation & $\mathrm{S}$ & $\begin{array}{l}\text { Note: Important for } \\
\text { Maintenance }\end{array}$ \\
\hline $\mathrm{H}-2-89443$ & $1-5$ & Inner/Outer Bellows Assembly & $\mathrm{S}$ & $\begin{array}{l}\text { Note: Important for } \\
\text { Maintenance }\end{array}$ \\
\hline $\mathrm{H}-2-89444$ & 1 & Cable Wiper Assembly & $\mathrm{G}$ & \\
\hline
\end{tabular}


HNF-2305

Rev. 0

Table 4.3 Core Sample Truck 2 Drawings

\begin{tabular}{|c|c|l|c|l||}
\hline Dwg. & Sht. & \multicolumn{1}{|c|}{ Description } & Cat. & Justification/Notes \\
\hline \hline H-2-89459 & $1-13$ & Alarm \& Control Diagram & E & $\begin{array}{l}\text {-Depicts Equipment } \\
\text { Essential to Safe Operation } \\
\text {-Depicts \& Identifies } \\
\text { Instrumentation Required } \\
\text { by AB }\end{array}$ \\
\hline H-2-89462 & 1 & Purge Gas Connection Box & G & \\
\hline H-2-89463 & $1-2$ & Purge Gas Cable Assembly & G & \\
\hline H-2-827187 & $1-8$ & $\begin{array}{l}\text { Electrical Truck 2 Connection } \\
\text { Diagram }\end{array}$ & E & $\begin{array}{l}\text { - Depicts Electrical Power } \\
\text { Diagram }\end{array}$ \\
\hline H-2-827191 & 1 & $\begin{array}{l}\text { Electrical Truck 2 One Line } \\
\text { Diagram }\end{array}$ & E & $\begin{array}{l}\text {-Depicts Electrical One- } \\
\text { line Diagram }\end{array}$ \\
\hline
\end{tabular}

\subsubsection{Summary of Core Sample Truck 2}

Table 4.3.1 Summary for Truck 2 Drawings

\begin{tabular}{||c|c|c||}
\hline \multicolumn{1}{|c|}{ Category } & Drawings & Sheets \\
\hline \hline General Drawings & 38 & 89 \\
\hline Support Drawings. & 16 & 87 \\
\hline Essential Drawings. & 6 & 34 \\
\hline
\end{tabular}


HNF-2305

Rev. 0

\subsection{CORE SAMPLE TRUCK 3 \& 4 DRAWINGS}

Table 4.4 RMCST $3 \& 4$ Drawings

\begin{tabular}{|c|c|l|c|l||}
\hline Dwg. & Sht. & \multicolumn{1}{|c|}{ Description } & Cat. & Justification/Notes \\
\hline \hline H-2-690000 & $1-2$ & RMCST Truck 3 \& 4 Assembly & S & \\
\hline H-2-690001 & $1-2$ & $\begin{array}{l}\text { RMCST Truck 3 \& 4 Drawing } \\
\text { Index }\end{array}$ & S & $\begin{array}{l}\text { Note: Important for } \\
\text { Maintenance }\end{array}$ \\
\hline H-2-690005 & $1-5$ & Drill Rig Equipment Installation & G & \\
\hline H-2-690006 & $1-3$ & Hydraulic Reservoir Assembly & G & \\
\hline H-2-690007 & $1-2$ & Pressure Indicator Light Assembly & G & \\
\hline H-2-690008 & $1-3$ & Hydraulic Flow Diagram & E & -Depicts Process Diagrams \\
\hline H-2-690009 & $1-2$ & Purge Gas Pneumatic Diagram & E & $\begin{array}{l}\text {-Depicts High Pressure } \\
\text { Process \& Instrumentation } \\
\text { Diagram }\end{array}$ \\
\hline H-2-690010 & $1-3$ & Truck Frame Assembly & G & \\
\hline H-2-690011 & $1-2$ & Center Outrigger Jack Assembly & G & \\
\hline H-2-690012 & $1-6$ & $\begin{array}{l}\text { Purge Gas Piping \& Hose } \\
\text { Installation }\end{array}$ & S & $\begin{array}{l}\text { Note: Important for } \\
\text { Maintenance }\end{array}$ \\
\hline H-2-690013 & $1-2$ & Auxiliary Air Supply Installation & G & \\
\hline H-2-690014 & $1-3$ & $\begin{array}{l}\text { Drill Head Hydraulic Piping } \\
\text { Installation }\end{array}$ & S & $\begin{array}{l}\text { Note: Important for } \\
\text { Maintenance }\end{array}$ \\
\hline H-2-690015 & $1-8$ & Hydraulic Controls Installation & S & $\begin{array}{l}\text { Note: Important for } \\
\text { Maintenance }\end{array}$ \\
\hline H-2-690016 & $1-3$ & Intercom System Installation & G & \\
\hline H-2-690017 & $1-2$ & Rear Jack Assembly & G & S \\
\hline H-2-690020 & $1-4$ & Shielded Receiver Assembly & $\begin{array}{l}\text { Note: Important for } \\
\text { Maintenance }\end{array}$ \\
\hline
\end{tabular}


HNF-2305

Rev. 0

Table 4.4 RMCST 3 \& 4 Drawings

\begin{tabular}{|c|c|c|c|c|}
\hline Dwg. & Sht. & Description & Cat. & Justification/Notes \\
\hline$H-2-690021$ & $1-3$ & $\begin{array}{l}\text { Line Conditioner \& Control } \\
\text { Electrical Panel Assembly }\end{array}$ & G & \\
\hline$H-2-690022$ & 1 & Shielded Receiver Tube Assembly & $\mathrm{G}$ & \\
\hline H-2-690023 & 1 & 3" Ball Valve Modification" & G & . \\
\hline H-2-690024 & 1 & Dust Cap and Sleeve Assembly & G & \\
\hline H-2-690025 & $1-2$ & View Port Shielded Receiver & G & \\
\hline $\mathrm{H}-2-690026$ & $1-2$ & $\begin{array}{l}\text { Shielded Receiver Electrical } \\
\text { Installation }\end{array}$ & $S$ & $\begin{array}{l}\text { Note: Important for } \\
\text { Maintenance }\end{array}$ \\
\hline $\mathrm{H}-2-690027$ & $1-2$ & Shielded Receiver Base Assembly & $\mathrm{G}$ & \\
\hline $\mathrm{H}-2-690028$ & $1-6$ & Shielded Receiver Weather Cover & G & \\
\hline $\mathrm{H}-2-690030$ & $1-4$ & Sampler Hoist Assembly & $S$ & $\begin{array}{l}\text { Note: Important for } \\
\text { Maintenance }\end{array}$ \\
\hline $\mathrm{H}-2-690031$ & $1-3$ & $\begin{array}{l}\text { Sampler Hoist Cable Feed } \\
\text { Assembly }\end{array}$ & G & \\
\hline$H-2-690032$ & 1 & $\begin{array}{l}\text { Sampler Hoist Cable Guide } \\
\text { Assembly }\end{array}$ & G & \\
\hline $\mathrm{H}-2-690033$ & $1-2$ & $\begin{array}{l}\text { Sampler Hoist Load Stand } \\
\text { Assembly }\end{array}$ & $\mathrm{G}$ & \\
\hline$H-2-690034$ & $1-4$ & Sampler Hoist Housing Assembly & G & \\
\hline H-2-690035 & $1-4$ & Breather Air Piping Installation & G & $\begin{array}{l}\text { Note: Installed but not } \\
\text { Used }\end{array}$ \\
\hline$H-2-690036$ & $1-2$ & $\begin{array}{l}\text { Sampler Hoist Cable Drum } \\
\text { Assembly }\end{array}$ & $\mathrm{G}$ & \\
\hline $\mathrm{H}-2-690037$ & 1 & Sampler Hoist Interlock Assembly & G & \\
\hline$H-2-690039$ & 1 & $\begin{array}{l}\text { Remote Latch Unit Weight } \\
\text { Assembly }\end{array}$ & G & \\
\hline
\end{tabular}


HNF-2305

Rev. 0

Table 4.4 RMCST 3 \& 4 Drawings

\begin{tabular}{|c|c|c|c|c|}
\hline Dwg. & Sht. & Description & Cat. & Justification/Notes \\
\hline $\mathrm{H}-2-690040$ & $1-5$ & RMCST Truck Bed Assembly & G & \\
\hline H-2-69004.1 & $1-4$ & $\begin{array}{l}\text { Rotary \& Stationary Platform Rail } \\
\text { Assembly }\end{array}$ & G & $\therefore$ \\
\hline $\mathrm{H}-2-690042$ & $1-2$ & Stationary Platform Assembly & G & \\
\hline$H-2-690043$ & 1 & $\begin{array}{l}\text { Shielded Receiver Lifting Frame } \\
\text { Installation }\end{array}$ & G & \\
\hline$H-2-690044$ & $1-2$ & $\begin{array}{l}\text { Shielded Receiver Lifting Frame } \\
\text { Assembly }\end{array}$ & G & \\
\hline $\mathrm{H}-2-690046$ & 1 & Lower Frame Assembly & G & \\
\hline H-2-690048 & 1 & Lower Bearing Mount Assembly & $\mathrm{G}$ & \\
\hline H-2-690049 & 1 & $\begin{array}{l}\text { Lower Frame \& Rotary Stop } \\
\text { Assembly }\end{array}$ & G & \\
\hline $\mathrm{H}-2-690050$ & $1-5$ & $\begin{array}{l}\text { Drillhead Service Platform } \\
\text { Installation }\end{array}$ & G & \\
\hline $\mathrm{H}-2-690051$ & $1-3$ & Quill Rod Assembly & G & Used as spare for Truck 2 \\
\hline $\mathrm{H}-2-690052$ & $1-2$ & Grapple Rotary Valve Actuator & G & \\
\hline $\mathrm{H}-2-690053$ & $1-4$ & Penetration Rate Assembly & G & \\
\hline$H-2-690054$ & $1-2$ & Rpm Sensing Ring Installation & G & \\
\hline $\mathrm{H}-2-690055$ & $1-5$ & Grapple Hoist Installation & $S$ & $\begin{array}{l}\text { Note: Important for } \\
\text { Maintenance }\end{array}$ \\
\hline H-2-690056 & $1-2$ & Grapple Hoist Mounting Support & G & \\
\hline H-2-690057 & $1-7$ & Grapple Hoist Assembly & $S$ & $\begin{array}{l}\text { Note: Important for } \\
\text { Maintenance }\end{array}$ \\
\hline$H-2-690058$ & $1-2$ & Grapple Hoist Housing Assembly & $\mathrm{G}$ & \\
\hline H-2-690059 & $1-6$ & Inner/Outer Bellows Assembly & $S$ & $\begin{array}{l}\text { Note: Important for } \\
\text { Maintenance }\end{array}$ \\
\hline
\end{tabular}


HNF-2305

Rev. 0

Table 4.4 RMCST 3 \& 4 Drawings

\begin{tabular}{|c|c|c|c|c|}
\hline Dwg. & Sht. & Description & Cat. & Justification/Notes \\
\hline $\mathrm{H}-2-690060$ & $1-4$ & Purge Gas Assembly & S & $\begin{array}{l}\text { Note: Important for } \\
\text { Maintenance }\end{array}$ \\
\hline $\mathrm{H}-2-690061$ & $1-4$ & Purge Gas Enclosure Assembly & G & \\
\hline $\mathrm{H}-2-690062$ & $1-5$ & Purge Gas Piping Assembly & S & $\begin{array}{l}\text { Note: Important for } \\
\text { Maintenance }\end{array}$ \\
\hline$H-2-690063$ & $1-3$ & Purge Gas Connection Box & G & \\
\hline H-2-690064 & $1-2$ & Purge Gas Cable Assembly & G & \\
\hline $\mathrm{H}-2-690065$ & 1 & Up Limit Switch Proximity Probe & G & \\
\hline$H-2-690066$ & $1-3$ & $\begin{array}{l}\text { Drillhead Service Platform } \\
\text { Assembly }\end{array}$ & G & \\
\hline H-2-690067 & $1-5$ & Drillhead Service Platform Assy & $\mathrm{G}$ & \\
\hline$H-2-690068$ & $1-7$ & Instrument Enclosure Assembly & $\mathrm{S}$ & $\begin{array}{l}\text { Note: Important for } \\
\text { Maintenance }\end{array}$ \\
\hline H-2-690069 & $1-18$ & Alarm \& Control Diagram & $\mathrm{E}$ & $\begin{array}{l}\text {-Depicts Required Alarm } \\
\text { Systems and Equipment } \\
\text { Essential to Safe Operation }\end{array}$ \\
\hline $\mathrm{H}-2-690070$ & $1-7$ & Elementary Diagram & E & $\begin{array}{l}\text {-Depicts Equipment } \\
\text { Essential to Safe Operation }\end{array}$ \\
\hline $\mathrm{H}-2-690071$ & $1-17$ & Electrical Connection Diagram & $S$ & $\begin{array}{l}\text { Note: Important for } \\
\text { Maintenance }\end{array}$ \\
\hline $\mathrm{H}-2-690073$ & $1-7$ & Electrical Installation & $S$ & $\begin{array}{l}\text { Note: Important for } \\
\text { Maintenance }\end{array}$ \\
\hline$H-2-690074$ & $1-2$ & $\begin{array}{l}\text { Hydraulic Bottom Detector Alarm } \\
\text { Panel Assembly }\end{array}$ & G & \\
\hline $\mathrm{H}-2-690076$ & $1-2$ & $\begin{array}{l}\text { Drill Rig Junction Box Enclosure } \\
\text { Assembly }\end{array}$ & $\mathrm{G}$ & \\
\hline
\end{tabular}


HNF-2305

Rev. 0

Table 4.4 RMCST 3 \& 4 Drawings

\begin{tabular}{||c|c|l|c|l||}
\hline Dwg. & Sht. & \multicolumn{1}{|c|}{ Description } & Cat. & Justification/Notes \\
\hline \hline H-2-690080 & $1-3$ & Rotary Platform Assembly & S & $\begin{array}{l}\text { Note: Important for } \\
\text { Maintenance }\end{array}$ \\
\hline H-2-690081 & $1-2$ & Rotary Platform Weldment & G & \\
\hline H-2-690082 & 1 & Bearing Plate Assembly & G & \\
\hline H-2-690083 & 1 & Rotary Stop Assembly & G & \\
\hline H-2-690084 & 1 & Slide Rail Details & G & \\
\hline H-2-690085 & 1 & $\begin{array}{l}\text { Control Console Support Frame } \\
\text { Assembly }\end{array}$ & G & \\
\hline H-2-690090 & $1-3$ & Control Console Assembly & G & \\
\hline H-2-690092 & $1-3$ & Motor Control Chassis Assembly & G & \\
\hline H-2-690094 & $1-2$ & Encoder Panel Assembly & G & \\
\hline H-2-690095 & $1-2$ & Loadcell Readout Assembly & G & \\
\hline H-2-690096 & $1-2$ & $\begin{array}{l}\text { Hydraulic \& Sampler Actuator } \\
\text { Control Panel }\end{array}$ & G & \\
\hline H-2-690097 & 1 & Panel Detail Circuit Breaker & G & \\
\hline H-2-690098 & 1 & Vent Panel Assembly & G & \\
\hline H-2-690106 & I-3 & Front Jack Assembly & G & \\
\hline H-2-690143 & $1-2$ & Mechanical Remote Latch Unit & S & $\begin{array}{l}\text { Note: Important for } \\
\text { Maintenance }\end{array}$ \\
\hline H-2-827188 & $1-7$ & $\begin{array}{l}\text { Electrical Truck 3 Connection } \\
\text { Diagram }\end{array}$ & E & $\begin{array}{l}\text {-Depicts Electrical Power } \\
\text { Diagrams }\end{array}$ \\
\hline H-2-827189 & $1-7$ & $\begin{array}{l}\text { Electrical Truck 4 Connection } \\
\text { Diagram }\end{array}$ & E & $\begin{array}{l}\text {-Depicts Electrical Power } \\
\text { Diagrams }\end{array}$ \\
\hline H-2-827192 & 1 & $\begin{array}{l}\text { Electrical Truck 3 One Line } \\
\text { Diagram }\end{array}$ & E & $\begin{array}{l}\text {-Depicts Electrical One- } \\
\text { line }\end{array}$ \\
\hline
\end{tabular}


HNF-2305

Rev. 0

Table 4.4 RMCST 3 \& 4 Drawings

\begin{tabular}{|c|c|l|c|l||}
\hline Dwg. & Sht. & \multicolumn{1}{|c|}{ Description } & Cat. & Justification/Notes \\
\hline \hline H-2-827193 & 1 & $\begin{array}{l}\text { Electrical Truck 4 One Line } \\
\text { Diagram }\end{array}$ & E & $\begin{array}{l}\text {-Depicts Electrical One- } \\
\text { line }\end{array}$ \\
\hline
\end{tabular}

\subsubsection{Summary RMCST 3 \& 4 Drawings}

Table 4.4.1 Summary RMCST 3 \& 4 Drawings

\begin{tabular}{||c|c|c||}
\hline \hline \multicolumn{1}{|c|}{ Category } & Drawings & Sheets \\
\hline General Drawings & 59 & 138 \\
\hline Support Drawings. & 18 & 94 \\
\hline Essential Drawings. & 8 & 46 \\
\hline
\end{tabular}

\subsection{CORE SAMPLING SUPPORT EQUIPMENT DRAWINGS}

Table 4.5 Core Sampling Support Equipment Drawings

\begin{tabular}{||c|c|l|c|l||}
\hline \hline \multicolumn{1}{|c|}{ Dwg. } & Sht. & \multicolumn{1}{|c|}{ Description } & Cat. & Justification/Notes \\
\hline \hline H-2-34727 & 1 & Core Sampling Cask & G & Not Used \\
\hline H-2-34994 & 1 & $\begin{array}{l}\text { Core Sampling Shipping Cask } \\
\text { Arrangement }\end{array}$ & G & Not Used \\
\hline H-2-34995 & 1 & $\begin{array}{l}\text { Core Sampling Shipping Cask } \\
\text { Detail }\end{array}$ & G & Not Used \\
\hline H-2-34996 & 1 & Core Sampling Shipping Cask & G & Not Used \\
\hline H-2-36057 & 1 & $\begin{array}{l}\text { Core Sampling Flapper } \\
\text { Assembly }\end{array}$ & G & Not Used \\
\hline H-2-36511 & 1 & Core Drill Pipe Fitting & G & Not Used \\
\hline
\end{tabular}


HNF-2305

Rev. 0

Table 4.5 Core Sampling Support Equipment Drawings

\begin{tabular}{|c|c|c|c|c|}
\hline Dwg. & Sht: & Description & Cat. & Justification/Notes \\
\hline $\mathrm{H}-2-36514$ & 1 & Core Sampling Seal & G & Not Used \\
\hline $\mathrm{H}-2-36766$ & 1 & $\begin{array}{l}\text { Core Sampling Equipment } \\
\text { Arrangement }\end{array}$ & G & Not Used \\
\hline $\mathrm{H}-2-36769$ & 1 & Core Sampling 12-in Flange & G & Not Used \\
\hline $\mathrm{H}-2-38078$ & 1 . & On-site Transfer Cask Stand & S & \\
\hline $\mathrm{H}-2-38079$ & $1-6$ & On-site Transfer Cask & $S$ & \\
\hline $\mathrm{H}-2-38191$ & 1 & $\begin{array}{l}\text { Arrangement and Detail Core } \\
\text { Sampler and Drill }\end{array}$ & $\mathrm{G}$ & \\
\hline $\mathrm{H}-2-38192$ & 1 & $\begin{array}{l}\text { Assembly and Detail Adapter } \\
\text { Flange Spray Washer Spray }\end{array}$ & G & \\
\hline $\mathrm{H}-2-38193$ & 1 & $\begin{array}{l}\text { Portable Core Sampling } \\
\text { Platform }\end{array}$ & $\mathrm{G}$ & \\
\hline H-2-38194 & 1 & Drip Pan for Core Sample & G & \\
\hline $\mathrm{H}-2-38195$ & 1 & $\begin{array}{l}\text { Commercial Drill Equipment } \\
\text { Mod }\end{array}$ & G & . \\
\hline H-2-38196 & 1 & $\begin{array}{l}\text { Misc Detail and Arrangement } \\
\text { for Core Sampling }\end{array}$ & G & . \\
\hline H-2-74814 & $1-5$ & Cask Truck & G & \\
\hline $\mathrm{H}-2-74815$ & 1 & Cask Truck & $\mathrm{G}$ & \\
\hline H-2-74816 & 1 & Cask Truck & G & \\
\hline $\mathrm{H}-2-74819$ & 1 & Cask Truck & $\mathrm{G}$ & \\
\hline $\mathrm{H}-2-74820$ & 1 & Cask Truck & $\mathrm{G}$ & \\
\hline $\mathrm{H}-2-79076$ & $1-2$ & Mobile Core X-Ray System & $\mathrm{G}$ & \\
\hline $\mathrm{H}-2-79840$ & 1 & Service Trailer Truck 1 & $\mathrm{G}$ & Not Used \\
\hline H-2-79841 & $1-3$ & Service Trailer Truck 1 & $\mathrm{G}$ & Not Used \\
\hline
\end{tabular}


HNF-2305

Rev. 0

Table 4.5 Core Sampling Support Equipment Drawings

\begin{tabular}{|c|c|c|c|c|}
\hline Dwg. & Sht. & Description & Cat. & Justification/Notes \\
\hline $\mathrm{H}-2-79842$ & $1-2$ & Service Trailer Truck 2 & G & \\
\hline $\mathrm{H}-2-79843$ & $1-2$ & Service Trailer Truck 2 & $\mathrm{G}$ & \\
\hline $\mathrm{H}-2-81842$ & $1-2$ & Cable Spray Washer Assy & G & \\
\hline $\mathrm{H}-2-81843$ & 1 & Cable Spray Washer Housing & G & \\
\hline $\mathrm{H}-2-81853$ & $1-6$ & Support Truck & G & \\
\hline $\mathrm{H}-2-83319$ & $1-2$ & Cask Truck, Unit 1 & G & \\
\hline $\mathrm{H}-2-83320$ & $1-4$ & Cask Truck, Unit 1 & G & \\
\hline $\mathrm{H}-2-83323$ & $1-2$ & Cask Truck Detail, Unit 2 & G & \\
\hline $\mathrm{H}-2-83324$ & $1-4$ & Unit 2 Cask Truck & G & \\
\hline $\mathrm{H}-2-83325$ & $1-5$ & Unit 1 Support Truck & G & \\
\hline $\mathrm{H}-2-85064$ & $1-3$ & $\begin{array}{l}\text { Sampler Valve Housing } \\
\text { Removal Tool }\end{array}$ & $\mathrm{G}$ & Used in Lab \\
\hline $\mathrm{H}-2-85066$ & $1-2$ & Changeout Valve & $\mathrm{G}$ & Not Used \\
\hline $\mathrm{H}-2-85287$ & $1-2$ & Mobile Air Charger Assembly & G & \\
\hline $\mathrm{H}-2-85299$ & 1 & $\begin{array}{l}\text { Nitrogen Trailer Hose Reel } \\
\text { Installation }\end{array}$ & $\mathrm{G}$ & \\
\hline $\mathrm{H}-2-85327$ & 4 & $\begin{array}{l}\text { Panelboard Schedule, Electrical } \\
\text { Distribution Trailer \#1 }\end{array}$ & $\mathrm{E}$ & $\begin{array}{l}\text { Already Essential (Other } \\
\text { sheets of this drawing are } \\
\text { NOT for sampling equip.) }\end{array}$ \\
\hline $\mathrm{H}-2-85327$ & 5 & $\begin{array}{l}\text { Panelboard Schedule, Electrical } \\
\text { Distribution Trailer \#2 }\end{array}$ & $\mathrm{E}$ & $\begin{array}{l}\text { Already Essential (Other } \\
\text { sheets of this drawing are } r \\
\text { NOT for sampling } \\
\text { equipment.) }\end{array}$ \\
\hline $\mathrm{H}-2-85340$ & 1 & $\begin{array}{l}\text { Distribution Trailer One-Line } \\
\text { Diagram }\end{array}$ & $\mathrm{E}$ & $\begin{array}{l}\text {-Depicts Electrical One- } \\
\text { line }\end{array}$ \\
\hline $\mathrm{H}-2-85341$ & 1 & Electrical Distribution Trailer \#2 & $\mathrm{G}$ & \\
\hline
\end{tabular}


HNF-2305

Rev. 0

Table 4.5 Core Sampling Support Equipment Drawings

\begin{tabular}{||c|c|l|c|l||}
\hline \multicolumn{1}{|c|}{ Dwg. } & Sht. & \multicolumn{1}{|c|}{ Description } & Cat. & Justification/Notes \\
\hline \hline H-2-85342 & 1 & Electrical Distribution Trailer \#1 & G & \\
\hline H-2-85345 & 1 & Core Barrel Assembly & G & Not Used \\
\hline H-2-85350 & $1-2$ & $\begin{array}{l}\text { Sampling Electrical Distribution. } \\
\text { Trailer }\end{array}$ & G & \\
\hline H-2-85358 & $1-4$ & Portable Platform Assembly & G & \\
\hline H-2-85601 & $1-2$ & $\begin{array}{l}\text { Breathing Air Compressor } \\
\text { Electrical One-Line Diagram }\end{array}$ & E & \\
\hline H-2-90257 & 1 & Core Sampler Latch Mechanism & G & Not Used \\
\hline H-2-91291 & $1-5$ & Sampler Hot Cell Unloader & G & Not Used \\
\hline H-2-91330 & 1 & $\begin{array}{l}\text { Pull Rod Magnetic Retreival } \\
\text { Tool }\end{array}$ & G & \\
\hline H-2-91331 & 1 & Magnetic Positioning Tools & G & \\
\hline H-2-91398 & 1 & Sampler Hot Cell Unloader & G & Not Used \\
\hline H-2-91422 & $1-2$ & Sampler Shoe Assembly & G & Not Used \\
\hline H-2-91425 & $1-2$ & Drill Bit Modification & G & Not Used \\
\hline H-2-91426 & 1 & Drill Bit Modification & G & Not Used \\
\hline H-2-91427 & $1-2$ & Drill Bit Plug Remover & G & Not Used \\
\hline H-2-91490 & $1-5$ & Sampler Hot Cell Unloader & G & Not Used \\
\hline H-2-91497 & $1-3$ & $\begin{array}{l}\text { Core Sampler Assembly and } \\
\text { Detail }\end{array}$ & G & Not Used \\
\hline H-2-91498 & $1-3$ & $\begin{array}{l}\text { Core Sampler Assembly and } \\
\text { Detail }\end{array}$ & G & Not Used \\
\hline H-2-91502 & 1 & $\begin{array}{l}\text { Core Catcher Assembly and } \\
\text { Detail }\end{array}$ & G & Not Used \\
\hline
\end{tabular}


HNF-2305

Rev. 0

Table 4.5 Core Sampling Support Equipment Drawings

\begin{tabular}{|c|c|c|c|c|}
\hline Dwg. & Sht. & Description & Cat. & Justification/Notes \\
\hline $\mathrm{H}-2-91503$ & $1-3$ & $\begin{array}{l}\text { Core Catcher Assembly and } \\
\text { Detail }\end{array}$ & G & Not Used \\
\hline $\mathrm{H}-2-91526$ & 1 & 12" Tank Riser Arrangement & G & Not Used \\
\hline $\mathrm{H}-2-91527$ & $1-2$ & 12" Drill Rod Guide & G & Not Used \\
\hline $\mathrm{H}-2-91539$ & 1 & 8" Tank Riser Arrangement & G & Not Used \\
\hline $\mathrm{H}-2-91540$ & $1-2$ & 8" Drill Rod Guide & $\mathrm{G}$ & Not Used \\
\hline H-2-91544 & 1 & 6" Tank Riser Arrangement & $\mathrm{G}$ & Not Used \\
\hline H-2-91545 & $1-2$ & 6" Drill Rod Guide & $\mathrm{G}$ & Not Used \\
\hline $\mathrm{H}-2-91546$ & 1 & $\begin{array}{l}12 " \mathrm{X} 6 " \text { Riser Adapter } \\
\text { Assembly }\end{array}$ & $\mathrm{G}$ & Not Used \\
\hline H-2-91549 & 1 & 4" Tank Riser Arrangement & $\mathrm{G}$ & \\
\hline $\mathrm{H}-2-91550$ & $1-2$ & 4" Drill Rod Guide & G & Not Used \\
\hline H-2-91551 & 1 & 12" X 4" Riser Adapter & $\mathrm{G}$ & Not Used \\
\hline $\mathrm{H}-2-91570$ & $1-2$ & Hot Cell Sample Removal Tool & $\mathrm{G}$ & Not Used \\
\hline $\mathrm{H}-2-91574$ & $1-3$ & Drill String Arrangement & G & \\
\hline $\mathrm{H}-2-91575$ & 1 & Core Barrel Assembly & G & Not Used \\
\hline H-2-91670 & 1 & $\begin{array}{l}\text { Drill Rodwasher Housing } \\
\text { Assembly and Details }\end{array}$ & G & \\
\hline H-2-91671 & $1-3$ & $\begin{array}{l}\text { Drill Rodwasher Manifold } \\
\text { Assembly and Details }\end{array}$ & G & \\
\hline H-2-91672 & 1 & Cover Plate Assembly & G & \\
\hline$H-2-91673$ & 1 & $\begin{array}{l}12^{\prime \prime} \mathrm{X} 18^{\prime \prime} \text { Riser Adapter } \\
\text { Assembly and Details }\end{array}$ & $\mathrm{G}$ & \\
\hline H-2-91674 & $1-3$ & $\begin{array}{l}\text { Transport Cask Stand Assembly } \\
\text { and Details }\end{array}$ & G & \\
\hline
\end{tabular}


HNF-2305

Rev. 0

Table 4.5 Core Sampling Support Equipment Drawings

\begin{tabular}{|c|c|c|c|c|}
\hline Dwg. & Sht. & Description & Cat. & Justification/Notes \\
\hline $\mathrm{H}-2-91675$ & 1 & $\begin{array}{l}\text { Cask Adapter Assembly and } \\
\text { Details }\end{array}$ & $\mathrm{G}$ & \\
\hline $\mathrm{H}-2-91676$ & 1 & PVC Sleeve Details & $\mathrm{G}$ & Not Used \\
\hline H-2-91677 & $1-2$ & $\begin{array}{l}\text { Kamlock Adapter Assembly: } \\
\text { Core Sampler Truck \#1 }\end{array}$ & $\mathrm{G}$ & \\
\hline $\mathrm{H}-2-91678$ & 1 . & Dust Cap Assembly & $\mathrm{G}$ & \\
\hline $\mathrm{H}-2-91679$ & $1-2$ & $\begin{array}{l}\text { Hoist Cable Spray Washer } \\
\text { Assembly }\end{array}$ & $\mathrm{G}$ & Not used \\
\hline $\mathrm{H}-2-91680$ & 1 & $\begin{array}{l}\text { Hoist Cable Washer Housing } \\
\text { Assembly }\end{array}$ & $\mathrm{G}$ & Not used \\
\hline $\mathrm{H}-2-91684$ & 1 & Modified Quadralatch & G & Not Used \\
\hline H-2-91685 & $1-2$ & 1" Rotary Sludge Sampler & $\mathrm{G}$ & Not Used \\
\hline $\mathrm{H}-2-91687$ & 1 & $\begin{array}{l}\text { Reusable Pull Rod Overpack } \\
\text { Assembly }\end{array}$ & $\mathrm{G}$ & Not Used \\
\hline $\mathrm{H}-2-94558$ & 1 & Sample Truck Hoist Trolley & $\mathrm{G}$ & \\
\hline $\mathrm{H}-2-99316$ & $1-4$ & Sludge Sampler $7 / 8$ & $\mathrm{G}$ & Not Used \\
\hline $\mathrm{H}-2-99332$ & 1 & $12^{\prime \prime} \times 4^{\prime \prime}$ Riser Adapter & $\mathrm{G}$ & \\
\hline $\mathrm{H}-2-99333$ & 1 & $12^{\prime \prime} \mathrm{X} 6$ " Riser Adapter & $\mathrm{G}$ & \\
\hline H-2-99334 & 1 & $12^{\prime \prime}$ X 8" Riser Adapter & $\mathrm{G}$ & \\
\hline $\mathrm{H}-2-99335$ & 1 & $12^{\prime \prime} \mathrm{X} 4$ " Riser Adapter & G & \\
\hline $\mathrm{H}-2-99336$ & 1 & $\begin{array}{l}6,8 \& 4^{\prime \prime} \times 4^{\prime \prime} \text { Offset Riser } \\
\text { Adapter }\end{array}$ & $\mathrm{G}$ & \\
\hline $\mathrm{H}-2-99347$ & 1 & $\begin{array}{l}6,8,10, \& 12^{\prime \prime} \text { Offset Riser } \\
\text { Adapter }\end{array}$ & $\mathrm{G}$ & \\
\hline $\mathrm{H}-2-99725$ & $1-5$ & Transfer Cask-NCAW & $\mathbf{S}$ & \\
\hline
\end{tabular}


HNF-2305

Rev. 0

Table 4.5 Core Sampling Support Equipment Drawings

\begin{tabular}{||c|c|l|c|c|c||}
\hline \multicolumn{1}{|c|}{ Dwg. } & Sht. & \multicolumn{1}{|c|}{ Deseription } & Cat. & Justification/Notes \\
\hline H-2-99727 & $1-2$ & Ebr-2 Storage Cask Modified & G & \\
\hline H-2-99729 & 1 & $\begin{array}{l}\text { Ebr-2 Cask/shield Receiver } \\
\text { Adapter }\end{array}$ & G & \\
\hline H-2-99730 & 1 & Drill String Burial Container & G & \\
\hline H-2-99731 & $1-2$ & $\begin{array}{l}\text { NCAW Transport Cask Lifting } \\
\text { Bail }\end{array}$ & G & \\
\hline H-2-99732 & 1 & Drill String Burial Container & G & \\
\hline H-2-99733 & $1-2$ & $\begin{array}{l}\text { Drill String Burial Container } \\
\text { Assembly }\end{array}$ & G & \\
\hline H-2-99734 & 1 & $\begin{array}{l}\text { Drill String Burial Container } \\
\text { Detail }\end{array}$ & G & \\
\hline H-2-99735 & 1 & $\begin{array}{l}\text { Drill String Burial Container } \\
\text { Detail }\end{array}$ & G & \\
\hline H-2-99736 & 1 & $\begin{array}{l}\text { Drill String Burial Container } \\
\text { Detail }\end{array}$ & G & \\
\hline H-2-99738 & $1-2$ & Cask Field Loading Stand & G & \\
\hline H-2-99739 & 1 & Handling Tools NCAW Cask & G & \\
\hline H-2-99740 & $1-2$ & Viewport Camera & G & \\
\hline H-2-99741 & 1 & $\begin{array}{l}\text { Drill String Burial Container } \\
\text { Support }\end{array}$ & G & \\
\hline H-2-99742 & 1 & Support Stand Detail & G & G & \\
\hline H-2-99743 & 1 & Support Stand Detail & G & \\
\hline H-2-99744 & 1 & Support Stand Detail & G & \\
\hline H-2-690045 & $1-4$ & Maintenance Platform & G & \\
\hline H-2-690124 & $1-2$ & Downforce Calibration Stand & \\
\hline
\end{tabular}


HNF-2305

Rev. 0

Table 4.5 Core Sampling Support Equipment Drawings

\begin{tabular}{|c|c|c|c|c|}
\hline Dwg. & Sht. & Description & Cat. & Justification/Notes \\
\hline H-2-690125 & $1-2$ & Grapple Hoist Test Stand & G & \\
\hline H-2-690127 & 1 & Misc Equipment & G & \\
\hline H-2-690128 & $1-2$ & Riser Sleeve Assembly & G & \\
\hline H-2-690130 & $.1-2$ & Int Drill Rod Removal Tool & G & \\
\hline $\mathrm{H}-2-690131$ & $1-2$ & $\begin{array}{l}\text { Riser Sleeve Spray Wash } \\
\text { Assembly }\end{array}$ & G & \\
\hline H-2-690132 & 1 & Riser Adapter Assembly & G & \\
\hline H-2-690133 & $1-2$ & Sampler Changeout Assembly & G & \\
\hline $\mathrm{H}-2-690134$ & $1-5$ & Drill String Arrangement & $\mathrm{E}$ & $\begin{array}{l}\text { - Depicts containment } \\
\text { boundaries }\end{array}$ \\
\hline H-2-690136 & $1-2$ & Offset Riser Adapter & G & \\
\hline H-2-690137 & 1 & Core Sampler Inserts & G & \\
\hline$H-2-690139$ & 1 & Casting Finger & G & \\
\hline $\mathrm{H}-2-690140$ & $1-6$ & Core Sampler Assembly & $S$ & $\begin{array}{l}\text { Note: Important for } \\
\text { Maintenance }\end{array}$ \\
\hline $\mathrm{H}-2-821457$ & $1-4$ & Drill String Arrangements & E & $\begin{array}{l}\text {-Depicts equipment } \\
\text { Essential to Safe Operation }\end{array}$ \\
\hline $\mathrm{H}-2-825312$ & 1 & $\begin{array}{l}\text { Transfer Cask Modifications and } \\
\text { Lifting Bail }\end{array}$ & G & \\
\hline $\mathrm{H}-2-826513$ & $1-2$ & Drill Rod Purge Installation & G & \\
\hline H-9-000174 & 1 & Purge Gas Trailer Assembly & $\mathrm{E}$ & $\begin{array}{l}\text { ECN adds drawing sheets } \\
\text { to show flow schematic } \\
\text { diagram for } \mathrm{N}_{2} \text { trailer }\end{array}$ \\
\hline H-14-021922 & $1-2$ & Breathing Air Compressor P\&ID & $\mathrm{E}$ & $\begin{array}{l}\text { - Process and } \\
\text { Instrumentation Diagram }\end{array}$ \\
\hline
\end{tabular}


HNF-2305

Rev. 0

\subsubsection{Summary for Core Sampling Support Equipment Drawings}

Table 4.5.1 Summary of Core Sampling Support Equipment

Drawings

\begin{tabular}{|l|c|c||}
\hline \multicolumn{1}{|c|}{ Category } & Drawings & Sheets \\
\hline General Drawings & 115 & 205 \\
\hline Support Drawings & 4 & 18 \\
\hline Essential Drawings & 7 & 17 \\
\hline
\end{tabular}

\subsection{RMCST SAMPLING EXHAUSTER DRAWINGS}

Table 4.6 RMCST Exhauster Drawings

\begin{tabular}{||c|c|l|c|l||}
\hline \hline \multicolumn{1}{|c|}{ Dwg. } & Sht. & \multicolumn{1}{|c|}{ Description } & Cat. & Justification/Notes \\
\hline \hline H-14-0030122 & $1-3$ & $\begin{array}{l}\text { Core Sampling Vent } \\
\text { Equipment }\end{array}$ & S & $\begin{array}{l}\text { Will be superseded by.new } \\
\text { drawings when } \\
\text { modification work is } \\
\text { complete }\end{array}$ \\
\hline H-14-100739 & $1-2$ & Exhauster Heater Assembly & G & $\begin{array}{l}\text { Will be superseded by new } \\
\text { drawings when } \\
\text { modification work is } \\
\text { complete }\end{array}$ \\
\hline H-14-100740 & $1-2$ & $\begin{array}{l}\text { Misc Detail RMCS Exhauster } \\
\text { C }\end{array}$ & G & \\
\hline H-14-100741 & 1 & Regulator Assembly & G & Not Used \\
\hline H-14-100742 & $1-2$ & Breather Vent Stack & G & \\
\hline H-2-818186 & $1-4$ & Exhauster Assembly & G & Used by AR Vault \\
\hline H-2-818188 & 1 & Plenum Assembly & G & \\
\hline H-2-818189 & $1-3$ & $\begin{array}{l}\text { RMCS External Skid } \\
\text { Electrical Details }\end{array}$ & G & Used by AR Vault \\
\hline
\end{tabular}


HNF-2305

Rev. 0

Table 4.6 RMCST Exhauster Drawings

\begin{tabular}{|c|c|c|c|c|}
\hline Dwg. & Sht. & Description & Cat. & Justification/Notes \\
\hline $\mathrm{H}-2-818201$ & $1-2$ & $\begin{array}{l}\text { RMCS Skid Electrical } \\
\text { Assembly }\end{array}$ & $\mathrm{G}$ & \\
\hline $\mathrm{H}-2-818202$ & $1-4$ & Misc Detail Exhauster & $\mathrm{G}$ & \\
\hline $\mathrm{H}-2-818204$ & 1 & Bottle Rack Assembly RMCS & $\mathrm{G}$ & Not used \\
\hline $\mathrm{H}-2-818205$ & 1 & $\begin{array}{l}\text { Pre Filter Stand Assembly } \\
\text { RMCS }\end{array}$ & $\mathrm{G}$ & Not used \\
\hline $\mathrm{H}-2-818206$ & $1-2$ & Lift Assembly RMCS & G & \\
\hline $\begin{array}{c}\mathrm{H}-2-821455 \\
.\end{array}$ & $1-5$ & RMCS Exhauster Assembly II & G & $\begin{array}{l}\text { Will be superseded by new } \\
\text { drawings when } \\
\text { modification work is } \\
\text { complete }\end{array}$ \\
\hline $\mathrm{H}-2-821456$ & $1-2$ & $\begin{array}{l}\text { RMCS Exhauster Skid } \\
\text { Assembly II }\end{array}$ & G & $\begin{array}{l}\text { Will be superseded by new } \\
\text { drawings when } \\
\text { modification work is } \\
\text { complete }\end{array}$ \\
\hline $\mathrm{H}-2-829081$ & $1-2$ & Overall Assy Exhauster C & $S$ & \\
\hline $\mathrm{H}-2-829082$ & $1-2$ & $\begin{array}{l}\text { Mechanical Installation } \\
\text { Exhauster C }\end{array}$ & S & \\
\hline $\mathrm{H}-2-829083$ & $1-3$ & $\begin{array}{l}\text { Stack Instrumentation } \\
\text { Exhauster C }\end{array}$ & S & \\
\hline H-2-829084 & $1-3$ & $\begin{array}{l}\text { Sampling Exhauster Heater } \\
\text { Assembly Exhauster C }\end{array}$ & $\mathrm{S}$ & \\
\hline $\mathrm{H}-2-829085$ & $1-3$ & Skid Assembly Exhauster C & $\mathrm{G}$ & \\
\hline $\mathrm{H}-2-829086$ & $1-4$ & $\begin{array}{l}\text { Instrument \& Control Panel } \\
\text { Assembly Exhauster C }\end{array}$ & S & $\begin{array}{l}\text { Demonstrates Compliance } \\
\text { with Laws \& Regulations }\end{array}$ \\
\hline $\mathrm{H}-2-829087$ & $1-5$ & $\begin{array}{l}\text { Stack Enclosure Assembly } \\
\text { Exhauster C }\end{array}$ & G & \\
\hline
\end{tabular}


HNF-2305

Rev. 0

Table 4.6 RMCST Exhauster Drawings

\begin{tabular}{|c|c|c|c|c|}
\hline Dwg. & Sht. & Description & Cat. & Justification/Notes \\
\hline $\mathrm{H}-2-829088$ & $1-2$ & $\begin{array}{l}\text { Record Sampler Enclosure } \\
\text { Assembly Exhauster C }\end{array}$ & G & \\
\hline $\mathrm{H}-2-829089$ & $1-2$ & $\begin{array}{l}\text { Cam Probe Enclosure } \\
\text { Assembly Exhauster C }\end{array}$ & $\mathrm{G}$ & \\
\hline $\mathrm{H}-2-829090$ & $1-2$ & $\begin{array}{l}\text { Lower Weather Shield } \\
\text { Assembly Exhauster C }\end{array}$ & G & \\
\hline $\mathrm{H}-2-829091$ & $1-2$ & $\begin{array}{l}\text { Upper Weather Shield } \\
\text { Assembly Exhauster C }\end{array}$ & G & \\
\hline $\mathrm{H}-2-829100$ & 1 & Drawing Tree Exhauster $\mathrm{C}$ & S & \\
\hline $\mathrm{H}-2-829105$ & $1-5$ & Electrical Installation & $S$ & \\
\hline $\mathrm{H}-2-829106$ & 1 & $\begin{array}{l}\text { Piping and Instrumentation } \\
\text { Diagram Exhauster C }\end{array}$ & E & \\
\hline $\mathrm{H}-2-829107$ & $1-2$ & $\begin{array}{l}\text { Variable Frequency Drive } \\
\text { Exhauster C }\end{array}$ & $\mathrm{G}$ & \\
\hline $\mathrm{H}-2-829108$ & $1-4$ & $\begin{array}{l}\text { Instrument Enclosure } \\
\text { Connection Diagram } \\
\text { Exhauster C }\end{array}$ & $S$ & \\
\hline $\mathrm{H}-2-829110$ & $1-3$ & $\begin{array}{l}\text { Exhauster Electrical } \\
\text { Interconnect Exhauster C }\end{array}$ & $\mathrm{E}$ & \\
\hline $\mathrm{H}-2-829111$ & $1-2$ & $\begin{array}{l}\text { Junction Box Detail } \\
\text { Exhauster C }\end{array}$ & $\mathrm{G}$ & \\
\hline $\mathrm{H}-2-829120$ & $1-2$ & Overall Assy Exhauster B & $S$ & \\
\hline H-2-829121 & $1-2$ & $\begin{array}{l}\text { Mechanical Installation } \\
\text { Exhauster B }\end{array}$ & $S$ & \\
\hline $\mathrm{H}-2-829122$ & $1-3$ & $\begin{array}{l}\text { Stack Instrumentation } \\
\text { Exhauster B }\end{array}$ & $S$ & \\
\hline
\end{tabular}


HNF-2305

Rev. 0

Table 4.6 RMCST Exhauster Drawings

\begin{tabular}{|c|c|c|c|c|}
\hline Dwg. & Sht. & Description & Cat. & Justification/Notes \\
\hline $\mathrm{H}-2-829123$ & $1-3$ & $\begin{array}{l}\text { Sampling Exhauster Heater } \\
\text { Assembly Exhauster B }\end{array}$ & $S$ & \\
\hline $\mathrm{H}-2-829124$ & $1-3$ & Skid Assembly Exhauster B & G & \\
\hline H-2-829125 & $1-4$ & $\begin{array}{l}\text { Instrument \& Control Panel } \\
\text { Assembly Exhauster B }\end{array}$ & $S$ & \\
\hline $\mathrm{H}-2-829126$ & $1-5$ & $\begin{array}{l}\text { Stack Enclosure Assembly } \\
\text { Exhauster B }\end{array}$ & G & \\
\hline $\mathrm{H}-2-829127$ & $1-2$ & $\begin{array}{l}\text { Record Sampler Enclosure } \\
\text { Assembly Exhauster B }\end{array}$ & G & \\
\hline $\mathrm{H}-2-829128$ & $1-2$ & $\begin{array}{l}\text { Cam Probe Enclosure } \\
\text { Assembly Exhauster B }\end{array}$ & G & ? \\
\hline $\mathrm{H}-2-829129$ & $1-2$ & $\begin{array}{l}\text { Lower Weather Shield } \\
\text { Assembly Exhauster B }\end{array}$ & G & \\
\hline $\mathrm{H}-2-829130$ & $1-2$ & $\begin{array}{l}\text { Upper Weather Shield } \\
\text { Assembly Exhauster B }\end{array}$ & G & \\
\hline $\mathrm{H}-2-829134$ & 1 & $\begin{array}{l}\text { Piping and Instrumentation } \\
\text { Diagram Exhauster B }\end{array}$ & $\mathrm{E}$ & \\
\hline $\mathrm{H}-2-829135$ & $1-5$ & $\begin{array}{l}\text { Electrical Installation } \\
\text { Exhauster B }\end{array}$ & $S$ & \\
\hline $\mathrm{H}-2-829136$ & $1-2$ & $\begin{array}{l}\text { Variable Frequency Drive } \\
\text { Exhauster B }\end{array}$ & G & \\
\hline $\mathrm{H}-2-829137$ & $1-4$ & $\begin{array}{l}\text { Instrument Enclosure } \\
\text { Connection Diagram } \\
\text { Exhauster B }\end{array}$ & S & \\
\hline $\mathrm{H}-2-829139$ & $1-3$ & $\begin{array}{l}\text { Exhauster Electrical } \\
\text { Interconnect Exhauster B }\end{array}$ & $\mathrm{E}$ & \\
\hline $\mathrm{H}-2-829140$ & $1-2$ & $\begin{array}{l}\text { Junction Box Detail } \\
\text { Exhauster B }\end{array}$ & G & \\
\hline
\end{tabular}


HNF-2305

Rev. 0

Table 4.6 RMCST Exhauster Drawings

\begin{tabular}{||c|c|l||c|l||}
\hline \hline Dwg. & Sht. & \multicolumn{1}{|c|}{ Description } & Cat. & Justification/Notes \\
\hline \hline H-2-829141 & 1 & Drawing Tree Exhauster B & S & Important to Maintenance \\
\hline
\end{tabular}

\subsubsection{Summary for RMCST Exhauster Drawings}

Table 4.6.1 Summary for RMCST Exhauster Drawings

\begin{tabular}{||l|c|c||}
\hline \multicolumn{1}{|c|}{ Category } & Drawings & Sheets \\
\hline General Drawings & 30 & 72 \\
\hline Support Drawings. & 17 & 51 \\
\hline Essential Drawings. & 4 & 8 \\
\hline
\end{tabular}

\subsection{OTHER SAMPLING EQUIPMENT DRAWINGS}

Table 4.7 Other Sampling Equipment Drawings

\begin{tabular}{||c|c|l|c|c||}
\hline \multicolumn{1}{|c|}{ Dwg. } & Sht. & \multicolumn{1}{|c|}{ Description } & Cat. & Justification/Notes \\
\hline \hline & & AUGER SAMPLING DRAWINGS & & \\
\hline H-2-79960 & $1-7$ & Surface Sampler Auger Assembly & S & \\
\hline H-2-826321 & $1-2$ & Auger Bit & S & \\
\hline & & GRAB SAMPLING DRAWINGS & & \\
\hline H-2-37844 & $1-3$ & $\begin{array}{l}\text { Instrumentation Miscelaneous } \\
\text { Installation Details }\end{array}$ & S & \\
\hline H-2-85602 & $1-5$ & Supernatant and Sludge Sampler & S & \\
\hline
\end{tabular}


HNF-2305

Rev. 0

Table 4.7 Other Sampling Equipment Drawings

\begin{tabular}{|c|c|c|c|c|}
\hline Dwg. & Sht. & Description & Cat. & Justification/Notes \\
\hline H-2-85645 & $1-2$ & $\begin{array}{l}\text { Tri-Pod and Bottle Hoist for } \\
\text { Supernatant and Sludge Sampler }\end{array}$ & $\mathrm{S}$ & \\
\hline \multirow[t]{3}{*}{$\mathrm{H}-2-826597$} & $1-2$ & $\begin{array}{l}\text { Table Assembly for Supernatant } \\
\text { and Sludge Sampler }\end{array}$ & $S$ & \\
\hline & & VAPOR SAMPLING DRAWINGS & & \\
\hline & & TYPE 3 & & \\
\hline $\mathrm{H}-2-815402$ & $1-2$ & $\begin{array}{l}\text { VSS Piping and Instrumentation } \\
\text { Diagram }\end{array}$ & $\mathrm{G}$ & Not Used \\
\hline H-2-815403 & 1 & VSS Mimic Panel Assemblies & $\mathrm{G}$ & Not Used \\
\hline $\mathrm{H}-2-815404$ & $1-2$ & VSS Control Rack Assemblies & $\mathrm{G}$ & Not Used \\
\hline $\mathrm{H}-2-815405$ & $1-2$ & VSS I/O Rack Module Assy & $\mathrm{G}$ & Not Used \\
\hline $\mathrm{H}-2-815406$ & 1 & VSS Drawing Index Tree & $\mathrm{G}$ & Not Used \\
\hline H-2-815407 & $1-3$ & $\begin{array}{l}\text { VSS Exterior Component Rack } \\
\text { Assemblies }\end{array}$ & $\mathrm{G}$ & Not Used \\
\hline $\mathrm{H}-2-815408$ & $1-19$ & VSS Control Diagram & $\mathrm{G}$ & Not Used \\
\hline $\mathrm{H}-2-815409$ & $1-3$ & $\begin{array}{l}\text { VSS Control Rack Interconnect } \\
\text { Diagram }\end{array}$ & $\mathrm{G}$ & Not Used \\
\hline $\mathrm{H}-2-815410$ & $1-2$ & $\begin{array}{l}\text { VSS I/O Rack Interconnect } \\
\text { Diagrams }\end{array}$ & $\mathrm{G}$ & Not Used \\
\hline $\mathrm{H}-2-815411$ & 1 & VSS Cabling Arrangement & G & Not Used \\
\hline $\mathrm{H}-2-815412$ & $1-3$ & $\begin{array}{l}\text { VSS Interior Interconnect } \\
\text { Diagram }\end{array}$ & $\mathrm{G}$ & Not Used \\
\hline $\mathrm{H}-2-815413$ & 1 & VSS Solid State Relay Panel Assy & $\mathrm{G}$ & Not Usied \\
\hline H-2-815414 & $1-5$ & VSS Facility Interior Electrical & $\mathrm{G}$ & Not Used \\
\hline H-2-815415 & 1 & VSS Cable Assemblies , & $\mathrm{G}$ & Not Used \\
\hline
\end{tabular}


HNF-2305

Rev. 0

Table 4.7 Other Sampling Equipment Drawings

\begin{tabular}{|c|c|l|c|l||}
\hline Dwg. & Sht. & \multicolumn{1}{|c|}{ Description } & Cat. & \multicolumn{1}{|c|}{ Justification/Notes } \\
\hline \hline H-2-815417 & 1 & $\begin{array}{l}\text { VSS Intrinsic Barrier Box } \\
\text { Assembly }\end{array}$ & G & Not Used \\
\hline H-2-815418 & 1 & $\begin{array}{l}\text { VSS Heat Control Elementary } \\
\text { Diagram }\end{array}$ & G & Not Used \\
\hline H-2-815419 & $1-6$ & VSS Mechanical Installation & G & Not Used \\
\hline & & \multicolumn{1}{|c|}{ TYPE 4 } & & \\
\hline H-2-825301 & 1 & $\begin{array}{l}\text { In-Situ Sample Head Assembly \& } \\
\text { Details }\end{array}$ & S & \\
\hline H-2-825313 & $1-7$ & $\begin{array}{l}\text { Vapor Sampling Cart Assembly \& } \\
\text { Details }\end{array}$ & S & \\
\hline H-2-825314 & $1-2$ & $\begin{array}{l}\text { Vapor Sampling Cart Electrical } \\
\text { Installation and Interconnect } \\
\text { Diagram }\end{array}$ & S & \\
\hline
\end{tabular}

\subsubsection{Summary for Other Sampling Equipment Drawings}

Table 4.7.1 Summary for Other Sampling Equipment Drawings

\begin{tabular}{||l|c|c||}
\hline \multicolumn{1}{|c|}{ Category } & Drawings & Sheets \\
\hline General Drawings & 17 & 54 \\
\hline Support Drawings. & 9 & 31 \\
\hline Essential Drawings. & 0 & 0 \\
\hline \hline
\end{tabular}


HNF-2305

Rev. 0

\subsection{SUMMARY}

The results of the drawing evaluation of Characterization sampling equipment are summarized in Table 5.1. The table shows both number of drawings and number of sheets.

Table 5.1 Drawing Evaluation Summary

\begin{tabular}{|c|c|c|c|}
\hline \multirow{2}{*}{ Equipment } & \multicolumn{3}{|c|}{ Drawing Category } \\
\hline & $\begin{array}{c}\text { General } \\
\text { (Dwgs/Sheets) }\end{array}$ & $\begin{array}{c}\text { Support } \\
\text { (Dwgs/Sheets) }\end{array}$ & $\begin{array}{c}\text { Essential } \\
\text { (Dwgs/Sheets) }\end{array}$ \\
\hline Truck 1 & $32 / 52$ & $14 / 49$ & $5 / 14$ \\
\hline Truck $1 \& 2$ & $28 / 46$ & $2 / 3$ & $0 / 0$ \\
\hline Truck 2 & $38 / 89$ & $16 / 87$ & $6 / 34$ \\
\hline Truck $3 \& 4$ & $59 / 138$ & $18 / 94$ & $8 / 46$ \\
\hline Support Equipment & $115 / 205$ & $4 / 18$ & $7 / 17$ \\
\hline Exhauster & $30 / 72$ & $17 / 51$ & $4 / 8$ \\
\hline $\begin{array}{l}\text { Other Sampling } \\
\text { Equipment }\end{array}$ & $17 / 54$ & $9 / 31$ & $0 / 0$ \\
\hline TOTALS & $319 / 656$ & $80 / 333$ & $30 / 119$ \\
\hline \multicolumn{2}{|c|}{$\begin{array}{l}\text { GRAND TOTAL of Characterization } \\
\text { Drawings (Dwgs/Sheets) }\end{array}$} & \multicolumn{2}{|c|}{$429 / 1108$} \\
\hline
\end{tabular}

Only two of the drawings that are identified as essential are currently marked "Essential Drawing". Therefore, when funding is available, the rest of the drawings need to be updated to comply with the designated category. When the drawings for Trucks 3 and 4 are updated, it may be necessary to establish unique drawings for each truck for most of the essential drawings. Truck 3 and 4 drawings may also require unique drawings for some of the next used on assembly drawings. 
HNF-2305

Rev. 0

\subsection{REFERENCES}

HNF, 1997, HNF-PRO-242, Rev. 1, Project Hanford Policy and Procedure System,

Engineering Drawing Requirements, Fluor Daniel Hanford, Inc., Richland, Washington

HNF, 1998, HNF-3240, Rev. 0, Characterization Equipment Essential Drawing Plan, Lockheed Martin Hanford Corporation, Richland, Washington.

Wilson, 1997, HNF-SD-WM-ETP-216, Rev. 1, Engineering Task Plan for Truck 3 \& 4 Drawing Compliance and Evaluation, Lockheed Martin Hanford Corporation, Richland, Washington 